\title{
Effects of Wharton's jelly-derived mesenchymal stem cells on neonatal neutrophils
}

\author{
This article was published in the following Dove Press journal: \\ Journal of Inflammation Research \\ 3I December 2014 \\ Number of times this article has been viewed
}

\section{Imteyaz Khan' \\ Liying Zhang ${ }^{2}$ \\ Moiz Mohammed' \\ Faith E Archer' \\ Jehan Abukharmah' \\ Zengrong Yuan ${ }^{2}$ \\ S Saif Rizvi' \\ Michael G Melek' \\ Arnold B Rabson ${ }^{1,2}$ \\ Yufang Shi ${ }^{2}$ \\ Barry Weinberger ${ }^{\prime}$ \\ Anna M Vetrano ${ }^{1,2}$}

'Department of Pediatrics, Division of Neonatology, Rutgers Robert Wood Johnson Medical School, ${ }^{2}$ Rutgers Child Health Institute of New Jersey, New Brunswick, NJ, USA
Correspondence: Barry Weinberger Division of Neonatology, Department of Pediatrics, Rutgers Robert Wood Johnson Medical School, I Robert Wood Johnson Place, New Brunswick, NJ 08903, USA

Tel +l 7322355684

Fax +I 7322356609

Email weinbebi@rwjms.rutgers.edu
Background: Mesenchymal stem cells (MSCs) have been proposed as autologous therapy for inflammatory diseases in neonates. MSCs from umbilical cord Wharton's jelly (WJ-MSCs) are accessible, with high proliferative capacity. The effects of WJ-MSCs on neutrophil activity in neonates are not known. We compared the effects of WJ-MSCs on apoptosis and the expression of inflammatory, oxidant, and antioxidant mediators in adult and neonatal neutrophils.

Methods: WJ-MSCs were isolated, and their purity and function were confirmed by flow cytometry. Neutrophils were isolated from cord and adult blood by density centrifugation. The effects of neutrophil/WJ-MSC co-culture on apoptosis and gene and protein expression were measured.

Results: WJ-MSCs suppressed neutrophil apoptosis in a dose-dependent manner. WJ-MSCs decreased gene expression of NADPH oxidase-1 in both adult and neonatal neutrophils, but decreased heme oxygenase- 1 and vascular endothelial growth factor and increased catalase and cyclooxygenase- 2 in the presence of lipopolysaccharide only in adult cells. Similarly, generation of interleukin- 8 was suppressed in adult but not neonatal neutrophils. Thus, WJ-MSCs dampened oxidative, vascular, and inflammatory activity by adult neutrophils, but neonatal neutrophils were less responsive. Conversely, Toll-like receptor-4, and cyclooxygenase- 2 were upregulated in WJ-MSCs only in the presence of adult neutrophils, suggesting an inflammatory MSC phenotype that is not induced by neonatal neutrophils.

Conclusion: Whereas WJ-MSCs altered gene expression in adult neutrophils in ways suggesting anti-inflammatory and antioxidant effects, these responses were attenuated in neonatal cells. In contrast, inflammatory gene expression in WJ-MSCs was increased in the presence of adult but not neonatal neutrophils. These effects should be considered in clinical trial design before WJ-MSC-based therapy is used in infants.

Keywords: inflammation, umbilical cord, apoptosis, neutrophil, mesenchymal stem cells

\section{Introduction}

Mesenchymal stem cells (MSCs) are multipotent progenitor cells that mediate immune tolerance in recipient hosts. These cells are commonly isolated from bone, blood, and adipose tissues, as well as from umbilical cord Wharton's jelly. They home to damaged tissues and contribute to their repair by secretion of cytokines, chemokines, and extracellular matrix proteins. ${ }^{1}$ Bone marrow-derived (BM)-MSCs, the most commonly studied MSCs, are also highly immunosuppressive both in vitro and in vivo in animal models., ${ }^{2,3}$ BM-MSCs modulate immune responses by dendritic cells, lymphocytes, neutrophils, and monocytes, in part through the release of cytokines and lipid mediators. ${ }^{4}$ These cells also inhibit apoptosis and reduce the formyl-methionyl-leucyl-phenylalanine-induced respiratory burst in neutrophils by constitutive release of interleukin (IL)-6. ${ }^{5}$ Some evidence 
suggests that immunosuppression by BM-MSCs is dependent on their expression of the adhesion molecules, ie, intercellular adhesion molecule-1 (ICAM-1) and vascular cell adhesion molecule-1, indicating that cell-to-cell contact is required. ${ }^{3}$ However, the mechanisms of immunosuppression by MSCs are not known.

MSCs may have therapeutic utility in neonates, who are susceptible to inflammatory diseases, such as bronchopulmonary dysplasia. However, the invasiveness of bone marrow aspiration and the age-dependent degradation in quantity and quality of BM-MSCs limit their clinical potential. Human umbilical cord Wharton's jelly (WJ) provides an alternative source of MSCs that exhibit high proliferative capacity and multidifferentiation potential. ${ }^{6}$ These cells are abundant and accessible, and when compared with MSCs from adult bone marrow or adipose tissue, WJ-MSCs are more robustly proliferative and immunosuppressive. ${ }^{7}$ Of note, these cells can be expanded from the umbilical cord at the time of birth, raising the possibility of autologous treatment of newborns.

Neutrophils are nonproliferative circulating phagocytes that produce reactive oxygen intermediates and inflammatory cytokines, and also promote vascular remodeling by secretion of angiogenic growth factors. ${ }^{8}$ However, prolonged activation of these cells following hypoxic or infectious insults has been implicated in chronic inflammatory diseases associated with alterations in tissue architecture, such as bronchopulmonary dysplasia. ${ }^{9}$ Current therapies to ameliorate neutrophil cytotoxicity have not been completely effective in treating or preventing these conditions. WJ-MSCs have been proposed as cell-based prophylaxis or treatment for neonatal inflammatory diseases. ${ }^{10}$ However, the effects of WJ-MSCs on neonatal neutrophil activity are not known.

In the current studies, we compared the dose-dependent effects of WJ-MSCs on apoptosis and inflammatory function in neonatal and adult neutrophils. We hypothesized that WJ-MSCs would downregulate expression of oxidant and inflammatory mediators, and increase antioxidant expression in neutrophils. Experiments were performed in the presence or absence of IL-6 or ICAM-1 (CD54) blocking antibodies to investigate the possibility that these effects are mediated by soluble mediators or cell contact, respectively. ${ }^{3,11}$

\section{Materials and methods}

\section{Reagents}

Dulbecco's Modified Eagle's Medium, phosphate-buffered saline, and bacterial lipopolysaccharide (serotype 0128:B12) were purchased from Sigma Chemical Company (St Louis, MO, USA). Dextran was from ThermoFisher (Fairlawn,
NJ, USA). Ficoll-Paque was from GE Healthcare (Piscataway, NJ, USA), and heat inactivated fetal bovine serum was from Biosera US (Kansas City, MO, USA). Annexin V-APC, 7-actinomycin D (7-AAD) and cytometric bead array flex sets were from BD Biosciences (San Jose, CA, USA). RNeasy RNA purification kits were purchased from Qiagen (Chatsworth, CA, USA). Primers for real time polymerase chain reaction were obtained from Integrated DNA Technologies, Inc. (Coralville, IA, USA) and Power SYBR green master mix from Applied Biosystems (Foster City, CA, USA). Anti-human IL-6 and anti-human CD54 (ICAM-1) were from eBioscience (San Diego, CA, USA).

\section{Subjects}

These studies were approved by the institutional review board of Robert Wood Johnson Medical School. Umbilical cord blood samples were collected at the time of delivery of healthy term newborns ( $\geq 37$ weeks gestation) by elective cesarean section prior to labor, with tissue-specific informed consent. Subjects with clinical evidence of chorioamnionitis, funisitis, or other perinatal infections were excluded. For comparison, neutrophils were isolated from peripheral venous blood of healthy adult volunteers.

\section{WJ-MSC isolation}

WJ-MSCs from nine infants were isolated by peeling the outer membrane of the umbilical cord and dissecting out the arteries and vein, as previously described. ${ }^{12} \mathrm{WJ}$ was cut into 1-3 $\mathrm{mm}$ pieces and placed in $10 \mathrm{~cm}$ tissue culture dishes. After drying (15 minutes), Dulbecco's Modified Eagle's Medium $+10 \%$ fetal bovine serum was added. Half of the medium was changed on day 5 , and all media were changed every 3 days subsequently. Adherent cells reached confluence after 10-12 days. Cells from each subject were treated as a new cell line and tested for purity and expression of characteristic MSC markers by flow cytometry. Similar to previous reports, ${ }^{1-3}$ all nine lines expressed CD105, CD73, and CD90, but not CD45, CD34, or CD31. All experiments were performed no later than passage five, to maintain consistency.

\section{Neutrophil isolation and co-culture}

Polymorphonuclear neutrophils (PMN) were isolated by dextran sedimentation, Ficoll gradient centrifugation, and hypotonic lysis. Cells were resuspended in Dulbecco's Modified Eagle's Medium containing 10\% fetal bovine serum $\left(1 \times 10^{6}\right.$ cells $\left./ \mathrm{mL}\right)$. Neutrophil suspensions were incubated with adherent WJ-MSCs in 24 -well plates at $37^{\circ} \mathrm{C}$, at WJ-MSC to neutrophil ratios of 1:20-1:640 for apoptosis 
studies and 1:20 for analysis of gene and protein expression. Cell viability (defined by absence of uptake of 7-AAD fluorescence dye) was $>97 \%$ in all experiments.

\section{Measurement of neutrophil apoptosis}

After 24 hours in co-culture with WJ-MSCs, neutrophils were washed out of the wells, pelleted, and resuspended in phosphate-buffered saline at $1 \times 10^{7}$ cells $/ \mathrm{mL}$. Triplicate aliquots of cells $(100 \mathrm{~mL})$ were then incubated with Annexin V-APC (1:20) and 7-AAD (1:10; 15 minutes, room temperature). The remaining cells were lysed for measurement of gene expression, as described below. Cells were analyzed by flow cytometry on a FACSArray Bioanalyzer (BD Biosciences). Neutrophils were identified based on forward scatter and side scatter characteristics, and all analyses were performed on this population. Viable neutrophils were defined as those not taking up either Annexin V or 7-AAD. Necrotic cells were defined as those taking up only 7-AAD, and apoptotic cells as those binding Annexin V. Quadrant boundaries were determined using unstained, as well as positive control cells stained with either 7-AAD or Annexin V-APC, and statistics were performed based on the relative cell numbers in these quadrants.

\section{Gene expression}

PMN were incubated with WJ-MSCs (WJ-MSC + PMN, 1:20) or medium control (PMN) for 24 hours, in the presence or absence of lipopolysaccharide $(1 \mu \mathrm{g} / \mathrm{mL})$ and antibodies to ICAM-1 or IL-6 $(1 \mu \mathrm{g} / \mathrm{mL})$. Neutrophils and WJ-MSCs were separated after co-culture by adherence. Nonadherent neutrophils were harvested from the co-culture supernatant, and their purity was confirmed by morphology. Viable WJ-MSCs were identified as adherent to the culture plate after two washes with phosphate-buffered saline. Absence of residual neutrophils and WJ-MSC morphology were confirmed by microscopy. Total RNA was extracted from neutrophils or WJ-MSCs, complementary DNA generated, and gene expression quantified by real-time polymerase chain reaction using RT2 qPCR master mix (Qiagen) and amplified using a Stratagene MX300p instrument, with $\beta$-actin as standard. Full-length coding sequences were obtained from GenBank ${ }^{\mathrm{TM}}$. Primers were designed using Primer Express software. Forward and reverse primers used were: NOX-1，5'-CCTTGCACCGGTCATTCTTT-3'

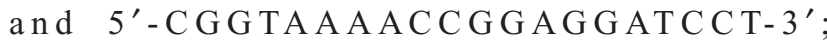
COX-2, 5' - GCC T GAT GAT T GCCCGAC T-3' and $5^{\prime}-\mathrm{GCTGGCCCTCGCTTATGATCT-3^{ \prime } \text { ; }}$ HO-1, 5'-GCTCAAAAAGATTGCCCAGA-3' and 5'-GCGGTAGAGCTGCTTGAACT-3'; catalase, 5'-CGGAGATTCAACACTGCCAA-3' and $5^{\prime}$ - GAAT GCCCGCACCT GAGTAA - 3'; VEGF, 5 '-GGCGTCGCACTGAAACTTTT-3' and 5' - T C C GAAGCGAGA ACAGCC-3'; TLR4, 5' - C CAGA GCCGCTGGTGTATC T- 3' and 5'-AACTGCCAGGTCTGAGCAATCT-3'; $\beta$-actin, 5' - A A A GACC T G TACGCCAACAC-3' and 5'-GTCATACTCCTGCTTGCTGAT-3'.

\section{Protein expression}

Supernatants from 24-hour cultures of neutrophils and/or WJMSCs were incubated with premixed flex set beads coated with antibodies to IL-8, macrophage inflammatory protein (MIP)-1 $\beta$, and ICAM-1 for 1 hour in 96-well filtration plates. Premixed phycoerythrin-labeled detection reagent was then added to the wells, and the plates incubated at room temperature in the dark for 2 hours. Bead/protein complexes were then washed and analyzed for fluorescence intensity using a BD FACSArray Bioanalyzer. Data were analyzed using BD FCAP software (version 2.0) with five parameter curve fitting. Measurements from co-cultures (observed) were compared with the sum of the measurements from cultures of neutrophils and WJ-MSCs alone (expected) and data are presented as the mean $\pm 95 \%$ confidence interval.

\section{Statistical analysis}

Data were analyzed using Statistica version 5.5 (StatSoft, Inc., Tulsa, OK, USA). The effects of treatments were compared pairwise by Student's $t$-test.

\section{Results}

WJ-MSCs suppressed neutrophil apoptosis in a dose-dependent manner (Figure 1). WJ-MSC to neutrophil ratios greater than 1:80 resulted in significant decreases in neutrophil apoptosis over 24 hours, in both adult and neonatal cells, with optimal efficacy at a WJ-MSC to neutrophil ratio greater than 1:40. In order to have enough cells for gene expression and protein assays, a ratio of 1:20 was chosen for all experiments. As previously reported, ${ }^{13}$ apoptosis was significantly reduced in neonatal neutrophils when compared with adult cells, but this difference was not sustained in the presence of WJ-MSCs. Incubation with IL-6 or ICAM-1 blocking antibodies did not affect apoptosis (not shown).

NADPH oxidase-1 (NOX-1) is a membrane-bound enzyme that is important in generating the superoxide anion that drives respiratory burst activity in neutrophils. We found that WJ-MSCs suppressed NOX-1 expression in both adult and neonatal neutrophils (Figure 2). WJ-MSCs also 


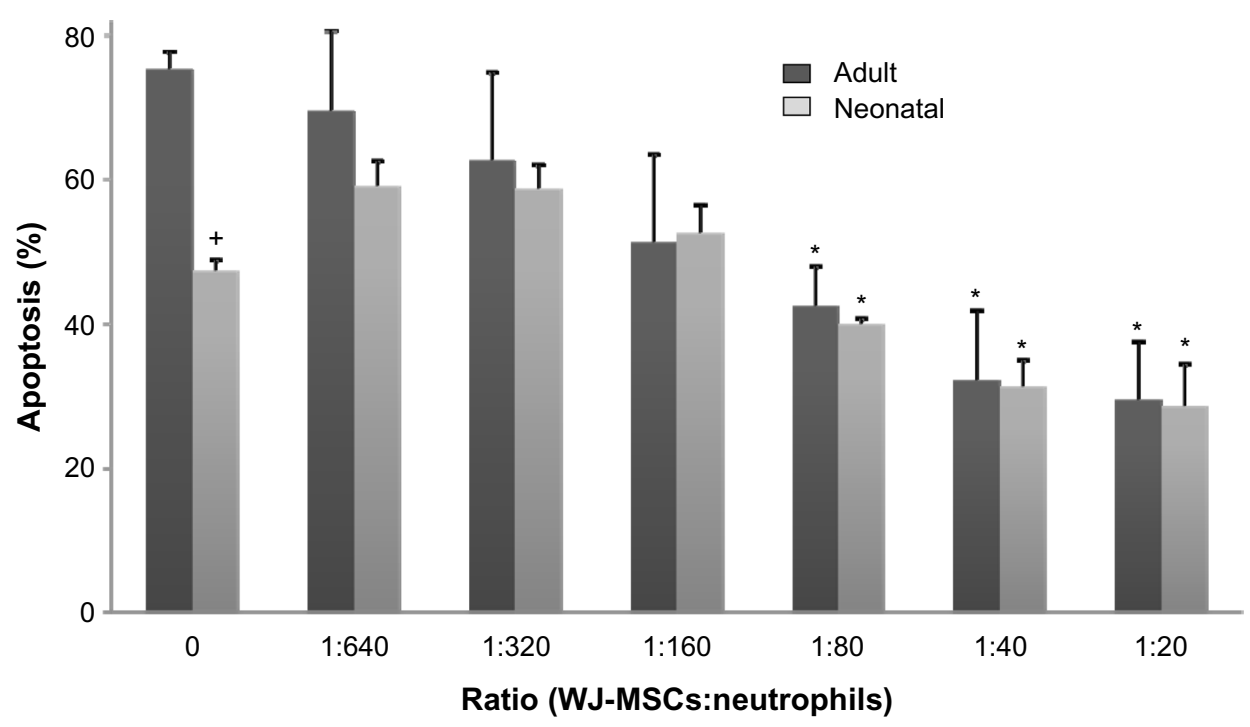

Figure I Effects of WJ-MSCs on neutrophil apoptosis.

Notes: Adult and neonatal neutrophils were incubated with WJ-MSCs at varying ratios for 24 hours and then analyzed for apoptosis by flow cytometry using BD FACSArray quadrant and two-dimensional histogram statistics based on relative fluorescence. Each bar represents the mean \pm standard error $(n=3-8)$. ${ }^{+}$ignificantly different $(P<0.05)$ from adult; *significantly different $(P<0.05)$ from untreated control $(0)$.

Abbreviation: MSCs, mesenchymal stem cells; WJ-MSCs, MSCs derived from Wharton's jelly.

markedly induced expression of the antioxidant enzyme catalase and suppressed expression of heme oxygenase-1 (HO-1) and vascular endothelial growth factor (VEGF), but these changes were significant only in adult cells. Effects of WJ-MSCs on neutrophil expression of the NOX-1 gene were decreased by antibodies to IL- 6 or ICAM-1, catalase gene by anti-ICAM-1 only, VEGF by anti-IL- 6 only, and HO- 1 by neither antibody.

Lipopolysaccharide induced Toll-like receptor-4 (TLR4) expression in adult but not neonatal neutrophils (Figure 3A). These responses were not affected by co-culture with WJMSCs. Some previous reports have suggested that WJ-MSCs may be distinguished from other MSCs by their hyporesponsiveness to lipopolysaccharide associated with low expression of TLR4. ${ }^{14}$ Consistent with this, we found that lipopolysaccharide did not induce TLR4 in WJ-MSCs (Figure 3B). In contrast, co-culture with adult neutrophils markedly increased TLR4 expression in WJ-MSCs. Cyclooxygenase-2 (COX-2), which catalyzes the production of inflammatory eicosanoids in neutrophils, is regulated in part by TLR $4 .{ }^{15}$ We found that lipopolysaccharide markedly induced COX-2 expression (20-fold) in adult neutrophils (Figure 3A). COX-2 expression in adult neutrophils further increased (60-fold) in the presence of both lipopolysaccharide and WJ-MSCs. As for TLR4, neonatal neutrophils were not affected by lipopolysaccharide and/or WJ-MSCs. While WJ-MSCs did not express COX-2 in response to lipopolysaccharide or to co-culture with neutrophils, the combination of lipopolysaccharide and adult neutrophils markedly induced generation of COX-2 (40-fold; Figure 3B).

Both neutrophils and WJ-MSCs constitutively produced IL-8 (Table 1). Consistent with previous reports, production of IL-8 was markedly decreased in neonatal neutrophils when compared with adult neutrophils. The combined generation of IL- 8 by neutrophils and WJ-MSCs in co-culture was significantly decreased in adult but not neonatal cells. In contrast, expression of MIP-1 $\beta$, another chemokine associated with neutrophil recruitment, by neutrophils and WJ-MSCs was not affected by co-culture of these cells. Expression of ICAM-1 by neutrophils and WJ-MSCs was also not significantly affected by co-culture.

\section{Discussion}

Our finding that WJ-MSCs suppress neutrophil apoptosis in a dose-dependent manner indicates that the physiologic effects of WJ-MSCs are similar in some respects to those demonstrated by BM-MSCs. Previous reports have shown that BM-MSCs protect adult neutrophils from apoptosis by triggering STAT-3 signaling ${ }^{5}$ or by decreasing expression of Bax, a proapoptotic member of the Bcl-2 family. ${ }^{16} \mathrm{BM}-\mathrm{MSCs}$ secrete mediators (VEGF, transforming growth factor- $\beta$ ) that promote fibroblast activation, angiogenesis, and alteration of tissue architecture, ie, processes that may be facilitated by neutrophils. ${ }^{17}$ Thus, prolonged viability of neutrophils in the presence of MSCs may facilitate MSC-mediated tissue repair. Our findings also suggest that the immunomodulatory 

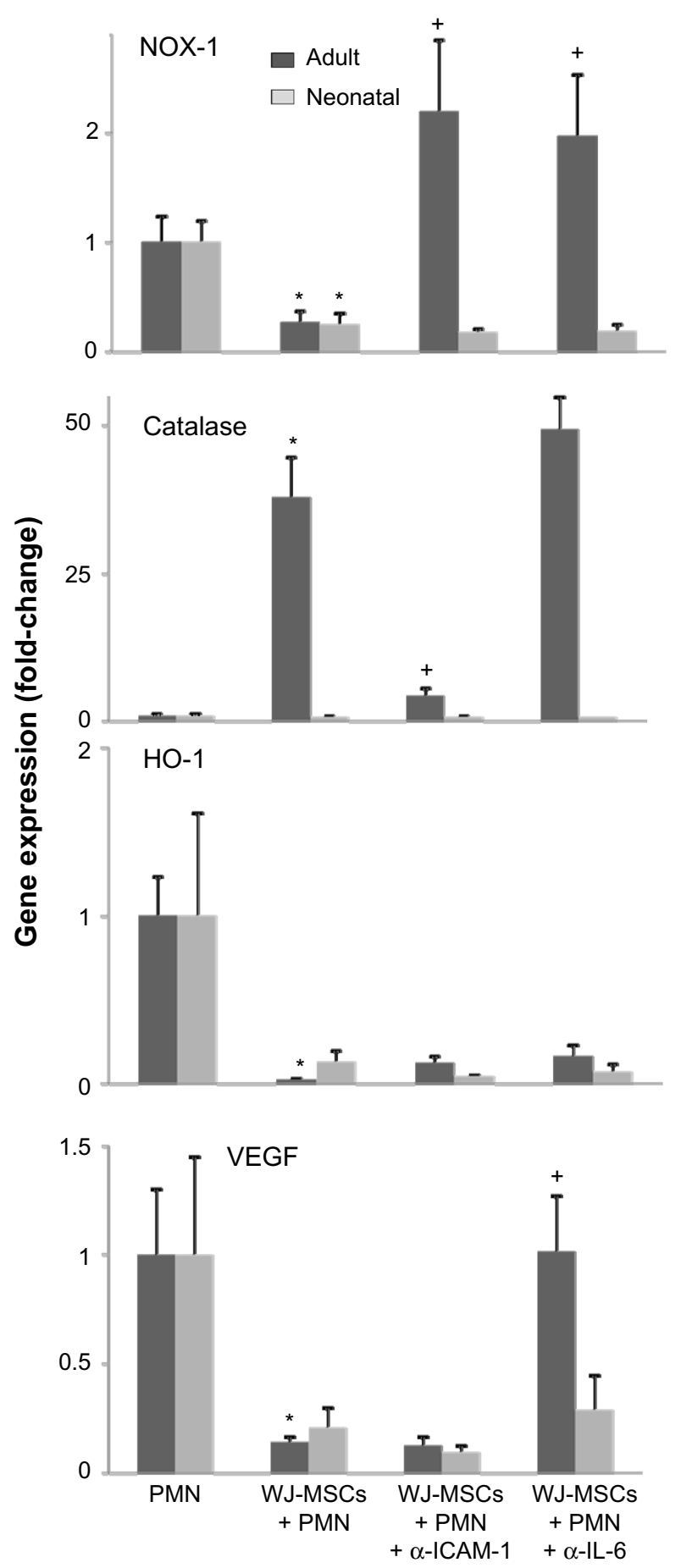

Figure 2 Expression of VEGF, catalase, HO-I, and NOX-I in neutrophils exposed to WJ-MSCs.

Notes: Adult and neonatal neutrophils were incubated with WJ-MSCs (WJ-MSCs + PMN, I:20) or medium control (PMN) for 4 hours, in the presence or absence of neutralizing antibodies to ICAM-I or IL-6 (I $\mu \mathrm{g} / \mathrm{mL})$. Total RNA was extracted, complementary DNA generated, and gene expression quantified by real-time polymerase chain reaction. Results were normalized to $\beta$-actin expression. Each bar represents the mean \pm standard error $(n=5-7)$. *Significantly different $(P<0.05)$ from control; 'significantly different $(P<0.05)$ from MSCs + PMN. Abbreviations: NOX-I, NADPH oxidase-I; HO-I, heme oxygenase-I; VEGF, vascular endothelial growth factor; MSCs, mesenchymal stem cells; WJ-MSCs, MSCs derived from Wharton's jelly; PMN, polymorphonuclear neutrophils; IL-6, interleukin-6; ICAM-I, intercellular adhesion molecule-I. effects of WJ-MSCs do not occur by facilitating neutrophil clearance, but most likely by decreasing their activity.

To investigate this, we next quantified the effects of WJ-MSCs on the expression of pro-oxidant and antioxidant enzymes in neutrophils. Neutrophils exert their bactericidal and inflammatory activity, in part, by the release of reactive oxygen intermediates, but prolonged neutrophil activity can cause cytotoxicity. ${ }^{18} \mathrm{NOX}-1$ is expressed in these cells, mediating the transfer of electrons to molecular oxygen to produce the superoxide anion. We found that WJ-MSCs suppressed NOX-1 activity in both adult and neonatal neutrophils, which may serve to modulate oxidative activity and ameliorate potential tissue injury. WJ-MSCs also induced expression of the antioxidant catalase, but only in adult neutrophils. Catalase protects cells from reactive oxygen intermediates by catalyzing the decomposition of hydrogen peroxide to water and oxygen, and its basal expression is decreased in neonatal neutrophils. Our observation that catalase is further upregulated in adult cells is consistent with previous reports that MSCs ameliorate hepatic injury by upregulating catalase expression in the liver. ${ }^{19}$ Upregulation of catalase may protect adults from neutrophil-mediated cytotoxicity in the presence of WJ-MSCs, but neonates appear to be resistant to this antioxidant effect.

WJ-MSCs also significantly decreased expression of the anti-inflammatory enzyme $\mathrm{HO}-1$ in adult neutrophils but not in neonatal cells. HO-1 exerts anti-inflammatory effects by upregulating IL-10 and IL-1R antagonist expression. ${ }^{20}$ This results in decreased neutrophil rolling and adhesion, as well as transmigration of these cells to inflammatory sites. Thus, the relative preservation of HO-1 activity in neonatal neutrophils exposed to WJ-MSCs may be a marker of preserved immune responses. Similarly, WJ-MSCs suppressed VEGF gene expression in adult, but not neonatal cells. VEGF is important in physiologic angiogenesis that drives tissue differentiation and growth. ${ }^{8}$ Taken together, the hyporesponsiveness of neonatal neutrophils suggests that interactions between WJ-MSCs and neutrophils in neonates may be characterized by sustained immune activity and ongoing tissue modeling and repair, whereas those in adult cells are predominantly characterized by suppression of oxidant activity and immunosuppression.

In contrast with earlier reports indicating that ICAM-1 is important in mediating the immunosuppressive effects of BM-MSCs, ${ }^{3}$ antibodies to ICAM-1 did not alter the effects of WJ-MSCs on apoptosis or expression of antioxidant or inflammatory mediators in neonatal neutrophils. 

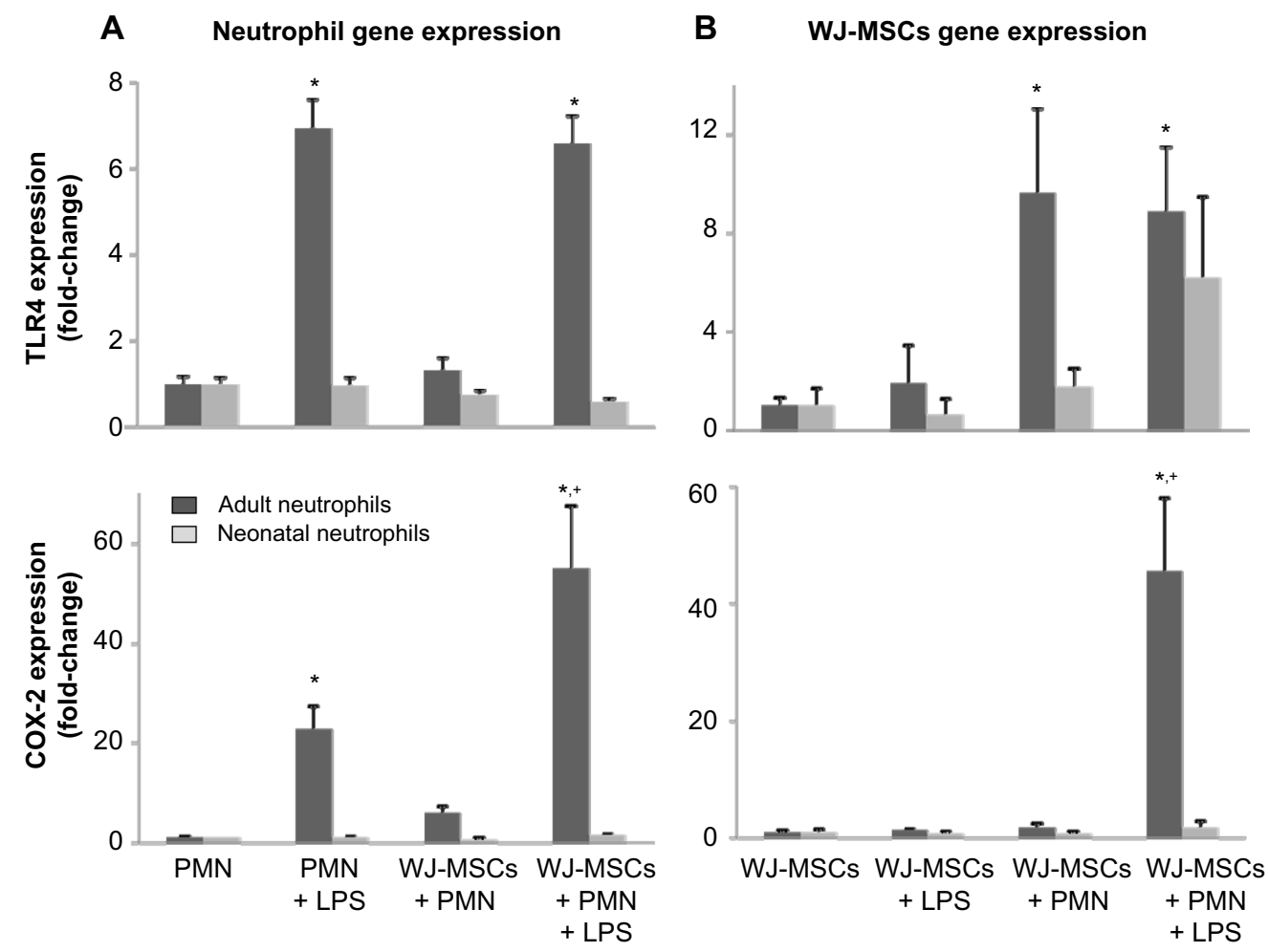

Figure 3 Expression of TLR-4 and COX-2 in neutrophils and WJ-MSCs.

Notes: (A) Adult and neonatal neutrophils were incubated in medium control (PMN), LPS (I $\mu \mathrm{g} / \mathrm{mL}$ ) and/or WJ-MSCs (WJ-MSCs to neutrophils, I:20) (B) WJ-MSCs were incubated in medium control (MSCs), with LPS (I $\mu \mathrm{g} / \mathrm{mL})$, and/or adult or neonatal neutrophils (WJ-MSCs to neutrophils, I:20). Total RNA was extracted from neutrophils (A) or WJ-MSCs (B), complementary DNA generated, and gene expression quantified by real-time polymerase chain reaction. Results were normalized to $\beta$-actin expression. Each bar represents the mean \pm standard error $(n=5-7)$. *Significantly different $(P<0.05)$ from PMN control; ${ }^{+}$significantly different $(P<0.05)$ from PMN + LPS $(\mathbf{A})$ or MSCs + LPS (B).

Abbreviations: COX-2, cyclooxygenase-2; MSCs, mesenchymal stem cells; WJ-MSCs, MSCs derived from Wharton's jelly; PMN, polymorphonuclear neutrophils; LPS, lipopolysaccharide; TLR4, Toll-like receptor-4.

Thus, ICAM-mediated cell contact is not likely an obligatory pathway for these effects. It is possible that this represents a mechanistic distinction between BM-MSCs and WJ-MSCs. Alternatively, our findings are consistent with previous studies reporting that the biologic effects of MSCs are mediated by soluble mediators, rather than direct contact. ${ }^{21,22}$ However, blocking antibodies to IL-6 also had no effect on WJ-MSC-mediated changes in neonatal neutrophil apoptosis or secretory activity. It is possible that MSCs exert paracrine effects via membrane-bound exosomes containing multiple soluble mediators affecting inflammatory and oxidative activity. ${ }^{23}$

TLR4 expression in neutrophils was upregulated by lipopolysaccharide in adult neutrophils, and this was not

Table I Production of inflammatory mediators by neutrophils and WJ-MSCs

\begin{tabular}{|c|c|c|c|c|}
\hline & $\begin{array}{l}\text { Neutrophils, } \\
\text { mean }(95 \% \mathrm{CI})\end{array}$ & WJ-MSCs mean $(95 \% \mathrm{CI})$ & $\begin{array}{l}\text { Neutrophil + WJ-MSCs } \\
\text { (expected) mean }(95 \% \mathrm{CI})\end{array}$ & $\begin{array}{l}\text { Neutrophil + WJ-MSCs } \\
\text { (observed) mean }(95 \% \mathrm{CI})\end{array}$ \\
\hline \multicolumn{5}{|l|}{ Adult } \\
\hline MIP-I $\beta(p g / m L)$ & $123(42,203)$ & $17(14,20)$ & $140(56,223)$ & $72(32,112)$ \\
\hline IL-8 (ng/mL) & $12(5,20)$ & $35(11,57)$ & $47(16,77)$ & $9(3,16)^{* *}$ \\
\hline ICAM-I (pg/mL) & $89(43,134)$ & $144(101,187)$ & $233(144,321)$ & $205(142,268)$ \\
\hline \multicolumn{5}{|l|}{ Neonatal } \\
\hline MIP-I $\beta(p g / m L)$ & $66(14,119)$ & $21(15,26)$ & $87(29,145)$ & $126(52,200)$ \\
\hline IL-8 (ng/mL) & $3(1,5)$ & $24(1,48)$ & $27(2,53)$ & $29(7,50)$ \\
\hline ICAM-I (pg/mL) & $102(46,157)$ & $143(50,236)$ & $230(96,393)$ & I $84(84,284)$ \\
\hline
\end{tabular}

Note: **Significantly different $(P<0.05)$ from neutrophil + WJ-MSCs (expected).

Abbreviations: CI, confidence interval; MSCs, mesenchymal stem cells; WJ-MSCs, MSCs derived from Wharton's jelly; IL, interleukin; ICAM-I, intercellular adhesion molecule-I; MIP-I $\beta$, macrophage inflammatory protein-I $\beta$. 
affected by WJ-MSCs. Previous studies have suggested that WJ-MSCs are distinct from BM-MSCs in that they do not express TLR4 and are nonresponsive to lipopolysaccharide. ${ }^{24}$ However, co-culture with adult neutrophils markedly upregulated expression of TLR4 in WJ-MSCs. This may represent a biologically relevant trigger for inflammation in adults, since TLR4-primed MSCs can differentiate into a phenotype ("MSC1") characterized by secretion of proinflammatory mediators. ${ }^{25}$ This pathway appears to be developmentally impaired in neonatal neutrophils, which do not upregulate TLR4 expression and do not induce it in WJ-MSCs. COX-2 expression is regulated in part by TLR $4 .{ }^{15} \mathrm{BM}-\mathrm{MSCs}$ bind lipopolysaccharide via surface TLR4 receptors, initiating a signaling cascade involving sequential activation of NF- $\kappa \mathrm{B}$ and upregulation of COX-2 production. ${ }^{26} \mathrm{We}$ found that gene expression of COX-2 by WJ-MSCs is markedly upregulated in the presence of adult neutrophils and lipopolysaccharide, but not of either of those alone. Similarly, COX-2 is induced in adult neutrophils by lipopolysaccharide, and significantly further increased by WJ-MSCs. Our findings suggest that WJ-MSCs and neutrophils generate prostaglandins or other eicosanoids under inflammatory conditions, but that these signaling pathways are developmentally impaired in neonates.

We also found that WJ-MSCs and adult neutrophils in co-culture generate significantly less IL- 8 than they do alone. IL-8 is a chemokine that activates neutrophil calcium mobilization and chemotaxis, ${ }^{27,28}$ so this may represent a mechanism for immunomodulation by WJ-MSCs. In contrast, WJ-MSCs did not affect the generation of ICAM-1 or the chemokine MIP-1 $\beta$ in either adult or neonatal neutrophils. ICAM-1 mediates transendothelial migration of neutrophils, and MIP-1 $\beta$ is secreted in large quantities by lipopolysaccharideactivated neutrophils. ${ }^{29}$ Our finding that WJ-MSCs do not affect expression of IL-8, ICAM-1, or MIP-1 $\beta$ in neonatal neutrophils suggests that WJ-MSCs may not suppress neutrophilic inflammation in this population as anticipated.

\section{Conclusion}

Hospitalized premature neonates are susceptible to diseases characterized by excessive inflammation, including bronchopulmonary dysplasia, and MSC-based therapies are thought to have potential in preventing or ameliorating these conditions. Animal studies of such therapies have shown efficacy in conditions including sepsis ${ }^{30}$ and acute lung injury, ${ }^{31}$ and initial trials in human neonates ${ }^{32}$ and adults ${ }^{33}$ have indicated feasibility and possible efficacy in bronchopulmonary dysplasia and chronic lung disease, respectively. In the current studies, WJ-MSCs suppressed apoptosis and dampened oxidative, vascular, and inflammatory activity by adult neutrophils, but neonatal neutrophils were less responsive. Conversely, TLR4 and COX-2 were upregulated in WJ-MSCs only when they were co-cultured with adult neutrophils, suggesting an inflammatory MSC phenotype that is not induced by neonatal neutrophils. Moreover, WJ-MSCs suppressed apoptosis of both adult and neonatal cells. In summary, WJ-MSCs may alter neutrophilic inflammation in adults and neonates via pathways that are distinct. These effects should be carefully considered and tested in clinical trials before WJ-MSC-based-therapy is promoted for prophylaxis or treatment in infants.

\section{Acknowledgment}

This research was supported by grants from the Gerber Foundation and the National Institutes of Health (R21ES021170, P30ES005022).

\section{Author contributions}

IK, BW and AMV, study design, acquisition of data, data analysis and drafting of the manuscript. LYZ, MM, JA, ZY and MGM, acquisition of data and critical review of the manuscript. FEA and SSR, acquisition of data, data analysis and critical review of the manuscript. ABR and YS, study design and critical review of the manuscript.

\section{Disclosure}

The authors report no conflicts of interest in this work.

\section{References}

1. Shi Y, Hu G, Su J, et al. Mesenchymal stem cells: a new strategy for immunosuppression and tissue repair. Cell Res. 2010;20:510-518.

2. Ren G, Su J, Zhang L, et al. Species variation in the mechanisms of mesenchymal stem cell-mediated immunosuppression. Stem Cells. 2009;27:1954-1962.

3. Ren G, Zhao X, Zhang L, et al. Inflammatory cytokine-induced intercellular adhesion molecule-1 and vascular cell adhesion molecule-1 in mesenchymal stem cells are critical for immunosuppression. J Immunol. 2010;184:2321-2328.

4. Matthay MA, Thompson BT, Read EJ, et al. Therapeutic potential of mesenchymal stem cells for severe acute lung injury. Chest. 2010;138: 965-972.

5. Raffaghello L, Bianchi G, Bertolotto M, et al. Human mesenchymal stem cells inhibit neutrophil apoptosis: a model for neutrophil preservation in the bone marrow niche. Stem Cells. 2008;26:151-162.

6. Zhou C, Yang B, Tian Y, et al. Immunomodulatory effect of human umbilical cord Wharton's jelly-derived mesenchymal stem cells on lymphocytes. Cell Immunol. 2011;272:33-38.

7. Kim DW, Staples M, Shinozuka K, Pantcheva P, Kang SD, Borlongan CV. Wharton's jelly-derived mesenchymal stem cells: phenotypic characterization and optimizing their therapeutic potential for clinical applications. Int J Mol Sci. 2013;14:11692-11712. 
8. Gaudry M, Bregerie O, Andrieu V, El Benna J, Pocidalo MA, Hakim J. Intracellular pool of vascular endothelial growth factor in human neutrophils. Blood. 1997;90:4153-4161.

9. Chess PR, D'Angio, Pryhuber GS, Maniscalco WM. Pathogenesis of bronchopulmonary dysplasia. Semin Perinatol. 2006;30:171-178.

10. Borghesi A, Cova C, Gazzolo D, Stronati M. Stem cell therapy for neonatal diseases associated with preterm birth. J Clin Neonatol. 2013;2:1-7.

11. Groppo R, Richter JD. CPEB control of NF-kappaB nuclear localization and interleukin-6 production mediates cellular senescence. Mol Cell Biol. 2011;31:2707-2714.

12. Bongso A, Fong CY. The therapeutic potential, challenges and future clinical directions of stem cells from the Wharton's jelly of the human umbilical cord. Stem Cell Rev. 2013;9:226-240.

13. Hanna N, Vasquez P, Pham P, et al. Mechanisms underlying reduced apoptosis in neonatal neutrophils. Pediatr Res. 2005;57:56-62.

14. Raicevic G, Najar M, Stamatopoulos B, et al. The source of human mesenchymal stromal cells influences their TLR profile as well as their functional properties. Cell Immunol. 2011;270:207-216.

15. Kirkby NS, Zaiss AK, Wright WR, et al. Differential COX-2 induction by viral and bacterial PAMPs: consequences for cytokine and interferon responses and implications for anti-viral COX-2 directed therapies. Biochem Biophys Res Commun. 2013;438:249-256.

16. Ottonello L, Frumento G, Arduino N, et al. Differential regulation of spontaneous and immune complex-induced neutrophil apoptosis by proinflammatory cytokines. Role of oxidants, Bax and caspase-3. J Leukoc Biol. 2002;72:125-132.

17. Hayashi Y, Tsuji S, Tsujii M, et al. Topical implantation of mesenchymal stem cells has beneficial effects on healing of experimental colitis in rats. J Pharmacol Exp Ther. 2008;326:523-531.

18. Hampton MB, Kettle AJ, Winterbourn CC. Inside the neutrophil phagosome: oxidants, myeloperoxidase, and bacterial killing. Blood. 1998;92:3007-3017.

19. Burra P, Arcidiacono D, Bizzaro D, et al. Systemic administration of a novel human umbilical cord mesenchymal stem cells population accelerates the resolution of acute liver injury. BMC Gastroenterol. 2012;12:88.

20. Piantadosi CA, Withers CM, Bartz RR, et al. Heme oxygenase-1 couples activation of mitochondrial biogenesis to anti-inflammatory cytokine expression. J Biol Chem. 2011;286:16374-16385.
21. Chen L, Tredget EE, Wu PY, Wu Y. Paracrine factors of mesenchymal stem cells recruit macrophages and endothelial lineage cells and enhance wound healing. PLoS One. 2008;3:e1886.

22. Gnecchi M, Zhang Z, Ni A, Dzau VJ. Paracrine mechanisms in adult stem cell signaling and therapy. Circ Res. 2008;103:1204-1219.

23. Lee C, Mitsialis SA, Aslam M, et al. Exosomes mediate the cytoprotective action of mesenchymal stromal cells on hypoxia-induced pulmonary hypertension. Circulation. 2012;126:2601-2611.

24. Tomchuck SL, Zwezdaryk KJ, Coffelt SB, Waterman RS, Danka ES, Scandurro AB. Toll-like receptors on human mesenchymal stem cells drive their migration and immunomodulating responses. Stem Cells. 2008;26:99-107.

25. Waterman RS, Tomchuck SL, Henkle SL, Betancourt AM. A new mesenchymal stem cell (MSC) paradigm: polarization into a proinflammatory MSC1 or an immunosuppressive MSC2 phenotype. PLoS One. 2010;5:e10088.

26. Tyndall A, Pistoia V. Mesenchymal stem cells combat sepsis. Nat Med. 2009; $15: 18-20$

27. Baggiolini M, Dewald B, Moser B. Interleukin-8 and related chemotactic cytokines - CXC and CC chemokines. Adv Immunol. 1994;55:97-179.

28. Zeilhofer HU, Schorr W. Role of interleukin-8 in neutrophil signaling. Curr Opin Hematol. 2000;7:178-182.

29. Issekutz AC, Rowter D, Springer TA. Role of ICAM-1 and ICAM-2 and alternate $\mathrm{CD} 11 / \mathrm{CD} 18$ ligands in neutrophil transendothelial migration. J Leukoc Biol. 1999;65:117-126.

30. Hall SR, Tsoyi K, Ith B, et al. Mesenchymal stromal cells improve survival during sepsis in the absence of heme oxygenase-1: the importance of neutrophils. Stem Cells. 2013;31:397-407.

31. Shalaby SM, El-Shal AS, Abd-Allah SH, et al. Mesenchymal stromal cell injection protects against oxidative stress in Escherichia coliinduced acute lung injury in mice. Cytotherapy. 2014;16:764-775.

32. Chang YS, Ahn SY, Yoo HS, et al. Mesenchymal stem cells for bronchopulmonary dysplasia: phase 1 dose-escalation clinical trial. J Pediatr. 2014;164:966-972.

33. Antunes MA, Laffey J G, Pelosi P, Rocco PR. Mesenchymal stem cell trials for pulmonary diseases. J Cell Biochem. 2014;115:1023-1032.
Journal of Inflammation Research

\section{Publish your work in this journal}

The Journal of Inflammation Research is an international, peer-reviewed open-access journal that welcomes laboratory and clinical findings on the molecular basis, cell biology and pharmacology of inflammation including original research, reviews, symposium reports, hypothesis formation and commentaries on: acute/chronic inflammation; mediators of inflamma-

\section{Dovepress}

tion; cellular processes; molecular mechanisms; pharmacology and novel anti-inflammatory drugs; clinical conditions involving inflammation. The manuscript management system is completely online and includes a very quick and fair peer-review system. Visit http://www.dovepress.com/ testimonials.php to read real quotes from published authors. 